\title{
EL «DRET REAL DEL VEDAT» EN LA BAILIA GENERAL DE ORIOLA Y ALACANT
}

\author{
Felipe Mateu y Llopis
}

Las presentes páginas quieren ser una breve exposición de lo que fue un impuesto, un «dret», en valenciano, que se percibía en la Bailla General de Orihuela y Alicante durante el régimen foral, y son además continuación de lo que se publicó en el número 4-5 de estos «Anales» (1985) sobre el «Dret real de Almoxarif».

La primera cuestión a considerar es la voz vedat; los diccionarios suelen definirla como vedado, campo o sitio acotado o cerrado por ley u ordenanza; así el Diccionario valenciano-castellano de José Escrig y Martínez en su tercera edición por Constantino Llombart (1887); la voz forma parte de la toponimia, comúnmente; amplían estas líneas los índices de mis Materiales para un Glosario de Diplomática Hispánica. Corona de Aragón. Reino de Valencia (Castellón, 1958) donde sólo se dio el concepto de «Coses vedades»; y los de la excelente obra Catálogo de la Exposición de Derecho Histórico del Reino de Valencia (1955); allí se relacionan diferentes impuestos, los Del General, los municipales y los reales; a estos últimos se refiere el del Vedat.

Los describe el Llibre de Capitols ab los quals se arrenden y Collecten los drets Real que te sa Magestat en la Governació y Batlia general de Oriola y Alacant, de Luis de Ocaña de 1613, Impres en Oriola per August Martinez a despeses de sa Magestat, citado.

En sus páginas 113 a 135 se insertan los Capitols ab los quals se arrenda el dret Real appellat del Vedat, en les ciutats de Oriola y Alacant, viles de Elig, Crevillent y Val de Elda y en tota la Batlia general deça Xexona. Como 
es sabido, la Bailía o Batlia deçà Xixona, era general, no local, pues que comprendía tierras varias de la «Governació general de la ciutat d'Oriola y Regne de Valencia», esto es, desde lo riu de Xixona hasta el límite de Murcia. Estos Capitols tienen, como los antecedentes, un gran interés lexicográfico, que tratamos de sintetizar, alfabéticamente.

Considerando la rareza del libro de Ocaña, de 1613, es útil lo que aquí se reproduce, por su valioso vocabulario; una breve relación de voces acredita la organización de la Bailia de Oriola: albaran de guia, aranzel, arrendador, arrendaments, Batle General, Batle local, collector, corredor, cort de la Batlia, dret de coses vedades, dret de la moneda, escrivà, lletres, macer, provisions reals, quintar esclaus, taula, taula ger, traure moneda, reals, recisio de contracte, Rey, salaris, verguer major, y otras voces de uso común.

Se hace referencia al privilegio de Fernando I, de 27 de octubre de 1407 que se ve en el Aureum Opus (fol. 171) dirigido Fidelibus nostris universis et singulis baiulis regni Valencie tam citra quam ultra sexonam vel eorum locatenentibus pressentibus et futuris, cuando el rey quería el officium baiulie generalis regni valencie supradicti ad ordinem antiquitus observatum, quod propter alienacionem et disipacionem nostri regii patrimonii factam per dictos reges progenitores nostros fuit et est totaliter perturbatum, lo que prueba el excelente gobierno de Fernando I el de Antequera (1412-1416), nieto del rey también organizador por excelencia Pedro el Ceremonioso, segundo de Valencia (1336-1387).

\section{LA REAL SENTENCIA DE 1573}

En la Declaració del Capitol / se trae «la sentencia Real donada en el S.S. Consell de Arago, entre parts de una lo Syndich de la ciutat de Alacant, y altra lo Procurador Fiscal y Patrimonial de sa Magestat», publicada en 10 de marzo de 1573, en la cual se hace referencia sobre el vectigal vulgo dictum, Lo dret Vedat, non solum de novem rebus expressis in foro Regis lacobi Secundi, aedito anno millesimo trecentessimo primo in lingua Valentina, Pegunta, Seu, Alquitra, fusta, Canem, ferro y armes e cavalls, sed etiam de aliis rebus, citadas en el Aranzel; a los referidos materiales y semovientes, cuya extracción estaba prohibida, se añadía el hilo de jarcia, los nueve conceptos señalados, en «les coses vedades».

Luego de las fórmulas de publicación se decía: «Aquesta Real sentencia com les demes que-s donen y pronuncien en lo S.S. Consell de Arago, fan ley en tot lo Regne, inter omnes, com está dispost per lo cap. 2 Et attento 
quod sententia, dels privilegis del Rey en loan, fol. 204», del Aureum Opus, dado en Valencia 30 de abril de 1438, en la página 467 de la edición de M.D. Cabanes Pecourt (1972), facsimilar reducida sobre la de 1515.

La Declaracio de com se executan y practiquen los Capitols ab los quals se arrenda lo dret Real del Vedat, en la Batlia general de Oriola, se inserta en los folios 115-116: «Aquest dret Real appellat del Vedat, se collecta en tots los llochs de la Batlia general deça Xexona, por compte de sa Magestat com per diverses lletres y provisions Reals, les quals estan insertades en lo primer llibre de lletres y provisions Reals recondit en lo Archiu de la Cort de la Batlia general de la ciutat de Oriola, la una de les quals es sa data en el Pardo a 16 de Novembre 1594 (fol. 501) y la altra es sa data en Toledo a 21 de Mars. 1600 (fol. 663) y los arrendaments de aquest dret se fan per lo Batle general y officials patrimonials en Oriola, y apres en Alacant, y no per altres Batles ni officials, y en lo primer arrendament que es fa en la ciutat de Oriola, entra la ciutat de Oriola y los llochs de ses hortes, que son Callosa, Coix, Granja, Albatera, Redovà, Catral, Almoradi, Daya, Guardamar, Rojals, Benetuzer, y en dita ciutat de Oriola se arrenda tambe ò es dona a collectar les Taules de Elig, y Crevillent, y de tota la Vall de Elda en la qual Vall entra Asp, Novelda, Elda, Petrel, Monover, y les Salines».

«Finits los Arrendaments dels drets Reals en dita ciutat de Oriola, los quals se fan segon dia de festes de la Nativitat del Señor, se va a Alacant, a arrendar los demes drets Reals de aquella ciutat hon lo Batle general y demes officials Patrimonials, arrenden dits drets los quals se collecten dels llochs de dites hortes, que son, Muchamel, S. Ioan, Benimagrell, Monfort y Palamo, los quals llochs son del terme y districte de dita ciutat. E ningun Batle Local de dita Batlia general te facultat de arrendar dits drets Reals ni de quintar Esclaus ni dar licencia de traure moneda, ni altres coses vedades ni prohibides fora Regne fora los Batles Locals de la ciutat de Oriola y Alacant, perço que en sos privilegis Reals tenen facultat, absent lo Batle general de fer tot ço que lo Batle general si present fosm.

\section{LA REAL PRAGMÁTICA DE 1599}

La Real Pragmática sanctio de la S.C.R. Magestat sobre la forma que se ha de guardar en despachar les licencies de les coses vedades y prohibides, traure fora lo Regne de 24 de diciembre de 1599, dada en Madrid y publicada en Valencia, es del tenor siguiente: «Ara ojats que us notifiquen y fan saber de part de la S.C.R. Majestat del Rey nostre señor e per aquella De part 
del Illustrissimo y Excellentissimo señor Don loan Alphonso Pimentel y de $\mathrm{He}$ rrera Compte de Benavent, Lloctinent y Capita general en la present ciutat y Regne de Valencia: Que per quant la Majestat del Rey nostre señor, nos es estada remesa una Real pragmatica sanctio fermada de la sua Real ma, y ab les solemnitats requisites y acostumbrades en deguda forma de Cancelleria despachada, manant que per nos sia feta publicar en la presente ciutat de Valencia, a fi y effecte que aquella sia ab tota puntualitat observada, y posat en execucio tot lo en ella per sa Majestat dispost y ordenat, segons en dita pragmatica Real es contengut, la qual es del serie y tenor seguent»:

«Nos D. Phelippe por la gracia de Dios Rey de Castilla, de Aragon, de Leon, de las Dos Sicilias, de Hierusalem, de Portugal, de Ungria, de Dalmacia, de Croacia, de Navarra, de Granada, de Toledo, de Valencia, de Galicia, de Mallorca, de Sevilla, de Cerdeña, de Cordova, de Corcega, de Murcia, de laen, de los Algarves, de Algezira, de Gibraltar, de las Islas de Canaria, de las Indias Orientales y Occidentales, Islas y tierra firme del mar Oceano, Archiduque de Austria, Duque de Borgoña, de Brabante, y de Milan, de Athenas y Neopatria, Conde Aspurg, de Flandes, de Tirol, de Barcelona, de Rossellon y Cerdaña, Marques de Oristan y Conde de Goceano».

«Por quanto por fueros, privilegios, y pragmaticas, del nuestro Reyno de Valencia esta prohibida la extraccion y saca de Pez, Sevo, Alquitran, madera, Cañamo, hilo de Jarcia, Hierro, Armas, Cavallos, Trigos, Cevadas, ganados, plata, cobre, cordovanes, brea, polvora, oro, y plata, monedado, y no monedado, arroz, daça, panizo, y otros por los mismos fueros, privilegios y pragmaticas del nuestro Reyno de Valencia prohibidas, por los mismos fueros y privilegios, en caso que se den y permita las licencias, y sacas, ha de ser graciosamente, y sin dinero alguno que por ellas se de y hemos entendido que esto no se guarda con la puntualidad que nos obligan los dichos fueros, y el juramento que de guardarlos tenemos prestado. Queriendo que se guarden a nuestros subditos y naturales del nuestro Reyno de Valencia sus fueros y libertades, por la presente nuestra pragmatica proveemos, estatuymos, mandamos y sancimos, por devida observancia de ellos, las cosas siguientes".

«Primeramente proveemos y mandamos que nos ni nuestro lugarteniente general, ni otro qualquier official nuestro por preeminente que sea, no podamos, ni puedan despachar semejantes gracias, y licencia por Secretaria, sino solamente por nuestra Real Chancilleria, ò de nuestro Lugarteniente general en el dicho Reyno de Valencia, y que las gracias y licencias en otra manera dadas, no tenga firmeza, ni tengan fuerça de licencia ni gracia, ni puedan ni devan ser executadas por ningun official nuestro, ni las personas en cuyo favor fueren dadas dichas licencias las puedan ni devan executar so 
pena a los officiales que las executaren de suspension de officio por un año, por la primera vez, y por la segunda dos, y por la tercera privacion de officio, y otras penas a nuestro arbitrio reservadas, y a la persona o personas en cuyo favor se dieren, si usaren dellas incurran en las mismas penas que incurriran e incurren si ficieren la tal extraccion sin nuestra licencia o de nuestro Lugarteniente general y otro official nuestro, y a mas desto en pena de otra tanta quantidad como fuere el valor de la mercaduria asi sacada aplicada a nuestro Fisco Real».

«Item estatuymos y ordenamos que por execucion de las dichas penas, se pueda proceder y proceda contra los assi delinquentes, y lo contrario facientes por Inquisicion mero officio a instancia de nuestro Real Fisco».

«ltem estatuymos y ordenamos que por las licencias y gracias que se despacharen assi por la nuestra Chancilleria que cabe nos reside, como por la de nuestro Lugarteniente General, no se pueda llevar ni lleve dinero alguno so color de trabajo, gratificacion, estrena, ò otro cualquier nombre cogitado, o incogitado, sino solamente la quantidad que por la expedicion, forma, registro, y sello, se puede y deve llevar, conforme a Reales ordinaciones de dicha Chancilleria. So pena que el official que tomare ultra lo dicho, cantidad alguna, incurra en privacion de officio en la forma susodicha, y el que diere la tal cantidad en pena de cinquenta libras moneda de Valencia por la primera vez, por la segunda cien libras, por la tercera incurra en pena de perdicion de la mercaduria que assi sacare, con el doble, aplicadas dichas penas a nuestro Real Fisco como dicho es y que se proceda y pueda proceder a Inquisicion de estos delitos y penas por mero officio, a instancia de nuestro Real Fisco.»

«ltem estatuymos y declaramos que en la dicha prohibicion y prohibiciones, no se entienda ser quitada la facultad a los Bayles generales en nuestro Reyno de Valencia, de poder dar dichas gracias y licencias de Pez, Sevo, Alquitran, Madera, Cañamo, hilo de jarcia, y Hierro, tan solamente en la forma que les esta concedida por los fueros del Reyno de Valencia, y estan en costumbre dello, quedando en todo lo demas las dichas prohibiciones en su fuerça efficacia y valor.»

Terminaba la Pragmatica de 1599 con las fórmulas de rigor, firmando el rey en el año "de todos nuestros Reynos el segundo", siendo Vicecanciller Covarruvias y publicada en Valencia en 29 de abril de 1600 por el Virrey Conde de Benavente.

Esta Pragmatica de 1599 fue confirmada y ampliada por otra dada por el mismo Felipe III en Aranda, el 14 de agosto de 1610, registrada in curia 
Valentiae, fol. 106, y mandada publicar por el virrey Marqués de Caracena en 7 de septiembre de dicho año; en ella se hace referencia a los Fueros de las últimas Cortes.

En la Declaracio del Capitol III se dice: «Notables. 1. Lo Collector o Arrendador del Dret Vedat no despacha sens precehit, licencia o ferma del Batle. En la Declaracio del IIII, «Notable: al Batle general se esguarda la administracio y lo arrendar los drets y rendes Reals privatives. Recuerda la obligada conexença y el privilegio del rey D. Fernando, 2 (cap. 7) en defensa de la regalía y añade: «Notables. 1. Lo Batle Local de Oriola absent lo General fa tot lo que el Batle general si present fos", aduciendo Regia sentencia (fol. 125) del Supremo Consell de Arago, de 1584. Menciona un Regio privilegio dado en Lisboa en 7 de febrero de 1582 y otro anterior dado en Madrid en 1577 sobre nombramiento de Don Juan Vich, como Baiulum generalem indicto nostro Valentiae Regno ultra Sexonam. El Sacrum Supremum Regium Concilium celebra sus sesiones en un aula del Palacio Real en la parroquia de San Gil, en 1585.

Al fin de la Declaracio del Capitol IIII concluye: "Lo Batle Local de Alacant te lo mateix exercici y facultat en dita ciutat y terme, y en tot son districte, absent lo Batle general no per sentencia, ni per privilegi, si per costum de temps que fonch creat dit offici de Batle Local de Alacant» (fol. 127v).

La del Capitol $V$ se refiere a una sentencia del Supremo Consell de Arago en duda sobre si la licencia de un antiguo arrendador del dret Real del Vedat, afectaba a uno nuevo. La Declaracio del VI, sobre el pago del dret de Almoxarif; la del VII sobre el Taulager del General qui dona albara de guia; la del VIII sobre el pago del dret de treta «que es degut en lo lloch de hon mou la cosa, y no en lo lloch per on ix, o es trau; remitiendo Vide Gutierrez, de Gabel, q.104 fol. 400 Ruberica quo in loco Gabella solvi debeat. an. 2.

Las Declaracions de los Capitols IX y X se refieren a lo dret Real de Almoxarif.

Memorial y Aranzel de les mercaduries que van fora lo Regne que de. huen Dret Vedat, en la Batlia General; son las siguientes:

Argent lo march, paga 1 sou. Azer lo quintar paga $2 \mathrm{~s}$. Aram lo quintar paga $2 \mathrm{~s}$. Alquitra lo quintar paga $1 \mathrm{~s}$. Agulles de Cozir lo miller paga 3 diners. Alabardes ab son ferro la dotzena paga $3 \mathrm{~s}$. Agulles de cap lo miller paga $2 \mathrm{~d}$. Ams de peixcar lo miller paga mig diner. Anells, o anelles de llauto la grossa, paga 6 d. Anelletes de cos de dona, la grossa, o miller, 2 d. Astes de llançes sens ferro, la dotzena paga $1 \mathrm{~s}$. 6 d. Arnes blanch paga $2 \mathrm{~s}$. Arnes 
de cama, o de cuixa, paga $6 \mathrm{~d}$. Adarga de fusta, paga $2 \mathrm{~d}$. Adarga de cuyro, paga $1 \mathrm{~d}$. Arbres de Ballesta per cascu paga 1 diner y mig. Artibanch paga $3 \mathrm{~d}$. Alesnes de Sabater, la dotzena, paga $1 \mathrm{~d}$. Archs la dotzena, paga $1 \mathrm{~s}$. Aros de torn, cascu, paga mig diner. Arcabuços falconetes cascu, paga $4 \mathrm{~d}$. Areners de llauto la dotzena, paga $2 \mathrm{~d}$. Areners de coure o altre metal la 12, paga $2 \mathrm{~d}$. Arros se despacha franchament.

Billo de Blanquers de Castella, lo quintar, paga 12 sous, ço es per dotze marchs de argent que hi ha en tot quintar, e mes per lo quintar de coure dos sous per tot. Ballesta cascuna paga $3 \mathrm{~d}$. Bras de Ballesta cascu, paga 1 diner y mig. Baciner de ferro, o azer, paga $6 \mathrm{~d}$. Broquers nous cascu paga $1 \mathrm{~d}$. Brides de Flandes la dotzena, paga $3 \mathrm{~d}$. Bota nova, paga $2 \mathrm{~d}$. Barrils nous lo parell, paga $3 \mathrm{~d}$. Bufet de noguer, o altra fusta gran, paga $8 \mathrm{~d}$. Bufet Chiquet, paga $4 \mathrm{~d}$.

Catiu Moro que va fora lo Regne paga 14 sous. Coure lo quintar, paga $2 \mathrm{~s}$. Caneles de Seu lo quintar, paga $2 \mathrm{~s}$. Seu lo quintar, paga $2 \mathrm{~s}$. Canems, o estopes de Canem, lo quintar paga $2 \mathrm{~s}$. Cardes o Carduçes lo parell, paga $1 \mathrm{~d}$. Cofre cada hu paga $3 \mathrm{~d}$. Cofrenet chich, paga, $1 \mathrm{~d}$. Cabaçet o Bovera, paga $1 \mathrm{~s}$. Caixa de fusta paga, $2 \mathrm{~d}$. Claus lo miller paga $2 \mathrm{~d}$. Cadira de fusta cascuna paga $1 \mathrm{~d}$. Curaça cascuna paga $1 \mathrm{~d}$. Cota de malla cascuna paga $1 \mathrm{~d}$. Cervellera ab galteres, paga $3 \mathrm{~d}$. Servellera sense galteres, paga $2 \mathrm{~d}$. Cancerros grans la dotzena, paga $1 \mathrm{~d}$.

Celada cascuna paga $1 \mathrm{~d}$. Capsanes cascuna paga $3 \mathrm{~d}$. Creuhera despasa cascuna, paga $1 \mathrm{~d}$. Cascabels de llauto la grossa, paga $1 \mathrm{~d}$. Cadenats chichs la dotzena, paga 1. Civelles o ferros de corretja la grossa, paga $3 \mathrm{~d}$. Carro nou cascu, paga 2 s. 6 d. Cubertas de cavall cascuna, paga, 1 d. Cint ab manera cascu, paga, $1 \mathrm{~d}$. Canfonia cascuna, paga $2 \mathrm{~d}$. Caixeta cascuna, paga, $1 \mathrm{~d}$. Ciulets la grossa, paga $1 \mathrm{~d}$. Crezols la dotzena, paga $1 \mathrm{~d}$. Costals de taules de Espaser cascu paga $1 \mathrm{~d}$. Capses la grossa cascuna paga $3 \mathrm{~d}$. Cadires de repos, grans cascuna paga 6 diners. Cadires de repos chiques cascuna, paga $3 \mathrm{~d}$. Corda o Cordell de canem lo quintar paga $2 \mathrm{~s}$.

Diners conforme al General per lliura paga $3 \mathrm{~d}$. Didals de llauto la grossa, paga $3 \mathrm{~d}$. Davant braços cascu paga $3 \mathrm{~d}$. Darts ab ferro la dotzena, paga $9 \mathrm{~d}$. Dogals de canem la dotzena, paga, $1 \mathrm{~d}$. Dogals de canem de cavall la dotzena paga $1 \mathrm{~d}$. y mig.

Estany lo quintar, paga 1 sou. Espases guarnides la dotzena paga $3 \mathrm{~s}$. Espasa la fulla paga $2 \mathrm{~d}$. Esperons la dotzena, paga $6 \mathrm{~d}$. Estelles de arch per obrar, la 12 paga 9 d. Elmet cascu paga 4 d. Espalaços cascu paga 4 d. Estoigs de Barber cascu paga $4 \mathrm{~d}$. Estoigs de dona cascu paga $4 \mathrm{~d}$. Esquelles 
grans la dotzena paga 2 d. Eixos de carro cascu paga 2 d. Espills de azer la dotzena paga $1 \mathrm{~d}$. Espills de fusta la dotzena paga $1 \mathrm{~d}$. Espills de azer grans la dotzena paga $2 \mathrm{~d}$. Espills de azer chichs la dotzena paga $1 \mathrm{~d}$. Espills de bori de fusta la dotzena paga $1 \mathrm{~d}$. Espingarda cascuna paga $4 \mathrm{~d}$. Espardeñes totes de canem la dotzena paga $2 \mathrm{~d}$. Espardeñes de sola despart y cara de canem, la dotzena de parells paga $1 \mathrm{~d}$. Esquelles chiques la dotzena paga $1 \mathrm{~d}$. Escriptori de fusta gran cascu paga $1 \mathrm{~s}$. Escriptori chiquet cascu paga $6 \mathrm{~d}$. Espaldar cascu paga $1 \mathrm{~s}$.

Frontilles la dotzena paga $2 \mathrm{~d}$. Flautes la grossa paga $1 \mathrm{~d}$. Flaviols chichs la grossa paga mig d. Ferro lo quintar paga $1 \mathrm{~s}$. Flaviols grans la grossa paga $1 \mathrm{~s}$. Fil de Ballesta lo quintar paga $2 \mathrm{~s}$. Fust de Ballesta per obrar la 12 paga $3 \mathrm{~d}$. Ferros de llances sens hastes la 12 paga $1 \mathrm{~s}$. $6 \mathrm{~d}$. Ferro de vires la grossa paga $6 \mathrm{~d}$. Ferro ab son faldo que es falda de malla cascu paga $6 \mathrm{~d}$. Fust appellat Carretol paga $2 \mathrm{~s}$. Fust appellat Doblera paga $1 \mathrm{~s}$. Fust appellat madero paga $3 \mathrm{~d}$. Fust de cardes la dotzena paga $1 \mathrm{~d}$. Fust de pintes de pentinar la 12 paga $1 \mathrm{~d}$. Fre cascu paga $1 \mathrm{~d}$. y mig.

Fussos la grossa paga $1 \mathrm{~d}$. Ferradures la dotzena paga $2 \mathrm{~d}$. Formes de çabata la dotzena paga 2 d. y mig. Fil de canem la quintar paga $2 \mathrm{~s}$.

Gavinets la dotzena paga $1 \mathrm{~d}$. Gafets la grossa paga $1 \mathrm{~d}$. Guaspes de espasa la grossa paga $3 \mathrm{~d}$. Guaspes chiques la grossa paga $2 \mathrm{~d}$. Glavis la dotzena paga $2 \mathrm{~s}$. Gofets cascu paga $3 \mathrm{~d}$. Guants de malla cascu paga 3 d. Guitarra cascuna paga $1 \mathrm{~d}$.

Lauto lo quintar paga $1 \mathrm{~s}$. Llançes la dotzena paga $3 \mathrm{~s}$. Llits de posts ab sos peus paga $8 \mathrm{~d}$. Lo Orgue paga $5 \mathrm{~s}$. $3 \mathrm{~d}$. Llit de fusta de camp paga 1 s. 4 d. Llit de fusta de cordes, cascu paga $8 \mathrm{~d}$. Llanternes grans de llanda o de ferro, la dotzena paga $2 \mathrm{~d}$. Llanternes chiques la dotzena paga $1 \mathrm{~d}$. Lauts cascu paga $1 \mathrm{~d}$.

Mantins la grossa paga $1 \mathrm{~d}$. Molla de restells per a fer torns cascu paga $4 \mathrm{~d}$. Manioples cascun parell paga $3 \mathrm{~d}$. Manacort cascu paga $1 \mathrm{~d}$. Mig cofre, cascu paga Mandrets cascun parell paga $2 \mathrm{~d}$. Metall obrat, o per obrar, lo quintar paga $2 \mathrm{~s}$. Martells de ferro grans la 12 paga $2 \mathrm{~d}$. Martells de ferro chichs, la 12 paga $1 \mathrm{~d}$.

Or per cada vint sous paga $3 \mathrm{~d}$. Perles y granats fins tres dines per lliura $3 \mathrm{~d}$. Pesga lo quintar paga $1 \mathrm{~s}$. Plom lo quintar paga $1 \mathrm{~s}$. Puñals la dotzena paga $1 \mathrm{~s}$. Pintes de pentinar lo cap lo miller paga $1 \mathrm{~s} .6 \mathrm{~d}$. Passadors la dotzena paga $1 \mathrm{~d}$. Pintes de fusta per a pentinar draps la dotzena paga $6 \mathrm{~d}$. Pintes de pentinar llana lo parell paga $1 \mathrm{~d}$. Pintes de fusta per a pentinar draps la 
dotzena paga 2 d. Pales de ferro la dotzena paga $2 \mathrm{~d}$. Poms cascu paga mig d. Paves grans cascu paga $3 \mathrm{~d}$. Paves chich cascu paga $2 \mathrm{~d}$. Plates cascuna paga $1 \mathrm{~d}$. Portadores cascun parell paga $3 \mathrm{~d}$. Pinçes la grossa cascuna paga $1 \mathrm{~d}$. Paternosters de fusta, cascuna grossa paga $1 \mathrm{~d}$. Polvara de Arcabus lo quintar paga $2 \mathrm{~s}$. Peto cascu paga $1 \mathrm{~s}$. Pedres fines, paga tres dines per lliura, $3 \mathrm{~d}$.

Restell cascu paga $2 \mathrm{~d}$. Rahors paga la dotzena $1 \mathrm{~s}$. Rems la dotzena paga $1 \mathrm{~s}$. 6 d. Salitre. Tacha lo miller paga $1 \mathrm{~d}$. Tenalles de ferro grans la 12 , paga $2 \mathrm{~d}$. Tenalles chiques, la dotzena paga $1 \mathrm{~d}$. Tisores de Abaixador, cascun parell paga $4 \mathrm{~d}$. Tisores de Sastre, o desquilar lo parell paga 1 diner $y$ mig. Tisores chiques, la dotzena paga $1 \mathrm{~d}$.

Telloles la dotzena paga $6 \mathrm{~d}$. Torn de filar llana paga $1 \mathrm{~d}$. Taula ab sos peus paga $3 \mathrm{~d}$. Tenalles la dotzena paga $1 \mathrm{~d}$. Tisores de Barreter lo parell paga $2 \mathrm{~d}$. Tiretes la grossa paga $1 \mathrm{~d}$. Talabarts la dotzena paga $1 \mathrm{~d}$. Telers de llana cascu paga $3 \mathrm{~d}$. Teler chich de llana de dona, paga $2 \mathrm{~d}$. Tonell de fusta cascu, paga $2 \mathrm{~d}$. Taula de fusta redona cascuna, paga $4 \mathrm{~d}$.

Viola cascuna paga $1 \mathrm{~d}$. Xaquimes de Mula de canem, la 12 paga $1 \mathrm{~d}$. Xaquimes de Cavall de canem, la dotzena paga $1 \mathrm{~d}$. y mig.

Este precioso arancel tiene múltiple valor, económico, lingüístico, mobiliario, para conocer desde el ajuar doméstico hasta el arnés del caballero.

\section{LUGARES EXENTOS}

«Aranzel de los llochs que no paguen dret de coses Vedades, exceptat lo dret de Pega per provisio Real. Son los seguents. Barcelona, Girona, Vila Franca. Palamos, Caller, Tortosa. Arago es franch de la mitat del dret de coses Vedades, exceptat Catiu Moro, que ha de pagar tot lo dret per entregue, que son 14 sous. Moltes altres coses y ha que dehuen lo dret de coses Vedades, que no estan continuadas en lo Aranzel, les quals son a arbitre del noble Batle eo Collector del dit Dret.

Totes les mercaduries que no dehuen dret ni obediencia axi que vagen fora lo Regne, com dins lo Regne, son:

Primo, tota manera de tintes, salvo Brazil y fustes; mes sendra Llombarde; mes tota cosa que proceheix de llana; mes tota cosa que proceheix de seda; mes tota manera de llensos; mes tota manera de cuyro no daurat; mes 
paper; mes fruyta verda; mes fil de çozir camises; mes qualsevol manera de vetes, que no tinguen or ni argent.

Cavall que entra en lo Regne y no torna a exir, no deu dret mes de llicencia. Freçes de cera llevada la mitat per la cera, paga per lo fil tres diners per arrova si va Arago, y si va a altra part fora lo Regne, paga sis diners per arrova. Pero de ordinari va esta mercaduria a Arago.

Fil de or que va fora lo Regne, se lleva la mitat por la seda, y per l'altra mitat paga $3 \mathrm{~d}$. per onça. E perque a les presents sis cartes la present compresa, y de ma propria scrites y tretes del memorial de les mercaduries que van fora lo Regne de Valencia que dehuen dret de la Batllia general y recondit en lo trast de hon se cobra lo dit dret en dita Cort de Batlia general de Valencia, plena fe hon se vulla y sia donada y atribuyda, yo Cristofol Maçana Notari en lloch e per lo Escriva de dita Cort assi pose mon Sig-nes (fol. 114-115).

Aquella organización de las Bailías, General y locales, que acredita el libro de Luis de Ocaña de 1613, sería continuada en aquel siglo y en el XVIII, como puede verse en la gran obra de Juan Bautista Vilar Orihuela, una ciudad valenciana en la España moderna. Historia de la Ciudad y Obispado de Orihuela. Prólogo de Sebastián García Martínez, publicada en 1981 por el Patronato Angel García Rogel creado en 1972 por la Caja de Ahorros de Nuestra Señora de Montserrate de Orihuela, Obra Social de la Caja de Ahorros de Alicante y Murcia. 